\title{
Hydration State Controls Stress Responsiveness and Social Behavior
}

\author{
Eric G. Krause, ${ }^{1}$ Annette D. de Kloet, ${ }^{1,2}$ Jonathan N. Flak, ${ }^{1,2}$ Michael D. Smeltzer, ${ }^{1}$ Matia B. Solomon, ${ }^{1}$ \\ Nathan K. Evanson, ${ }^{3}$ Stephen C. Woods, ${ }^{1}$ Randall R. Sakai, ${ }^{1}$ and James P. Herman ${ }^{1}$ \\ ${ }^{1}$ Department of Psychiatry and Behavioral Neuroscience, University of Cincinnati, College of Medicine, Cincinnati, Ohio 45219, ${ }^{2}$ Program in Neuroscience, \\ University of Cincinnati, Cincinnati, Ohio 45237, and ${ }^{3}$ Cincinnati Children's Hospital Medical Center, Cincinnati, Ohio 45229
}

Life stress frequently occurs within the context of homeostatic challenge, requiring integration of physiological and psychological need into appropriate hormonal, cardiovascular, and behavioral responses. To test neural mechanisms underlying stress integration within the context of homeostatic adversity, we evaluated the impact of a pronounced physiological (hypernatremia) challenge on hypothalamic-pituitary-adrenal (HPA), cardiovascular, and behavioral responses to an acute psychogenic stress. Relative to normonatremic controls, rats rendered mildly hypernatremic had decreased HPA activation in response to physical restraint, a commonly used rodent model of psychogenic stress. In addition, acute hypernatremia attenuated the cardiovascular response to restraint and promoted faster recovery to prestress levels. Subsequent to restraint, hypernatremic rats had significantly more c-Fos expression in oxytocin- and vasopressin-containing neurons within the supraoptic and paraventricular nuclei of the hypothalamus. Hypernatremia also completely eliminated the increased plasma renin activity that accompanied restraint in controls, but greatly elevated circulating levels of oxytocin. The endocrine and cardiovascular profile of hypernatremic rats was predictive of decreased anxiety-like behavior in the social interaction test. Collectively, the results indicate that acute hypernatremia is a potent inhibitor of the HPA, cardiovascular, and behavioral limbs of the stress response. The implications are that the compensatory responses that promote renal-sodium excretion when faced with hypernatremia also act on the nervous system to decrease reactivity to psychogenic stressors and facilitate social behavior, which may suppress the anxiety associated with approaching a communal water source and support the social interactions that may be encountered when engaging in drinking behavior.

\section{Introduction}

Stress, broadly defined as a real or perceived threat to homeostasis, activates neural circuits that alter the body's physiology and behavior to ensure survival and well-being. Physical stressors are real threats that create signals within the internal environment that communicate deviations from homeostasis to the brain. In contrast, perceived threats or psychogenic stressors create signals that arise within the brain itself as it interprets stimuli in the external environment as potential insults. Although from different origins, physical and psychogenic stressors activate overlapping hypothalamic circuitry mediating reactive responses. As an example, increased plasma tonicity (hypernatremia) is a physical stressor that stimulates osmoreceptors that communicate hydromineral imbalance and ultimately activate magnocellular neurons in the hypothalamic paraventricular nucleus (PVN),

Received Nov. 20, 2010; revised Feb. 14, 2011; accepted Feb. 15, 2011.

Author contributions: E.G.K., A.D.d.K., J.N.F., M.D.S., M.B.S., and J.P.H. designed research; E.G.K., A.D.d.K., J.N.F., M.D.S., M.B.S., N.K.E., R.R.S., and J.P.H. performed research; E.G.K. contributed unpublished reagents/analytic tools; E.G.K. analyzed data; E.G.K., S.C.W., and J.P.H. wrote the paper.

This work was supported by National Institutes of Health (NIH) Grant HL096830 (E.G.K.), American Heart Association Grant 09PRE2250169 and NIH Grant F31 NS068122 (A.D.d.K.), NIH Grant DK66596-05A2S1 (R.R.S.), NIH Grant DK66596 (R.R.S.), and NIH Grant MH49698 (J.P.H.).

The authors declare no competing financial interests.

Correspondence should be addressed to Dr. Eric G. Krause at the above address. E-mail: krauseeg@ucmail.uc.edu. DOI:10.1523/JNEUROSCI.6078-10.2011

Copyright $\odot 2011$ the authors $\quad 0270-6474 / 11 / 315470-07 \$ 15.00 / 0$ which coordinate autonomic and endocrine responses that work to restore body fluid homeostasis (Bourque, 2008; Toney and Stocker, 2010). Restraint (confinement in a small space, considered a "psychogenic stressor") activates parvocellular neurons within the PVN, governing autonomic function and endocrine release to cope with perceived trauma (Ulrich-Lai and Herman, 2009). Thus, real or perceived threats, although arising from different origins, descend on common hypothalamic circuits that coordinate compensatory responses aimed at protecting the body.

Because both kinds of stressors activate common hypothalamic circuits, it is reasonable that the two should interact (i.e., physical stressors may alter the perception, and consequently the impact, of temporally contiguous psychogenic stressors). For example, hypernatremia generates physiological signals that converge on the CNS to elicit corrective responses at the vasculature and kidneys, and at the same time they contribute to the perception of thirst, which in turn, promotes water-seeking and drinking behavior (Stricker and Sved, 2000). Central drive of these motivated behaviors may well influence the perception of psychogenic stressors that may be subsequently encountered. Moreover, the same autonomic and endocrine responses aimed at restoring hydromineral balance may also influence neural circuits regulating responding to psychogenic stress.

The brain coordinates responses to physical and psychogenic stressors and orchestrates appropriate neuroendocrine, autonomic, and behavioral responses (Ulrich-Lai and Herman, 2009). The pro- 
totypical stress response is elevated circulating glucocorticoids, which result from activation of the hypothalamic-pituitary-adrenal axis (HPA) (de Kloet, 2008). Additionally, stress often creates an abrupt shift in autonomic tone toward increased sympathetic and decreased parasympathetic activity (Sgoifo et al., 1999). Ultimately, physical or psychogenic stressors promote changes in behavior [e.g., hypernatremia induces water intake and restraint induces anxietylike behavior (Chotiwat and Harris, 2006; Krause et al., 2008)].

The overall goal of the present study was to determine the importance of feedback regarding physiological adversity on subsequent stress responsivity and behavior. Rats were rendered hypernatremic by acute administration of $2.0 \mathrm{M} \mathrm{NaCl}$ and subsequently underwent tests of HPA activation, cardiovascular reactivity, and social interaction. We found that neuroendocrine and autonomic mechanisms compensating for hydromineral imbalance may also act on the nervous system to limit responding to psychogenic stress and promote social interactions, thereby facilitating access to a limited environmental resource.

\section{Materials and Methods}

Animals. Adult male Sprague Dawley rats (Harlan) weighing 250-300 g at the initiation of the study arrived at least 2 weeks before the onset of the experiment and were individually housed on a $12 \mathrm{~h}$ light/dark (6:00 A.M. to 6:00 P.M.) schedule with ad libitum access to pelleted rat chow (Harlan Teklad) and water, except where otherwise noted. All procedures were approved by the University of Cincinnati Institutional Animal Care and Use Committee.

Procedure. At 10:00 A.M., water bottles were removed from cages, and immediately afterward, rats were injected subcutaneously with $1 \mathrm{ml}$ of 0.15 or $2.0 \mathrm{M} \mathrm{NaCl}$; each injection was preceded by a lidocaine injection into the same area of skin to minimize pain and irritation. One hour later, animals were killed and trunk blood samples were taken to measure pre-restraint levels of plasma renin activity (PRA) ( $n=4$ per group), oxytocin (OT) ( $n=5-6$ per group), vasopressin (AVP) $(n=4$ per group), plasma proteins ( $n=3$ per group), and hematocrit ( $n=3$ per group). Another cohort of rats was treated identically (i.e., water bottles removed and rats administered 0.15 or $2.0 \mathrm{M} \mathrm{NaCl}$ ), but $1 \mathrm{~h}$ after the injections were placed into plastic restrainers. Cohorts of these rats were killed, and trunk blood collected at 15, 30, or $60 \mathrm{~min}$ after the onset of restraint. Specifically, samples were collected for PRA and OT at 15, 30, 60 min after the onset of restraint ( $n=5-7$ per group per time point). Samples were collected for AVP at $15 \min (n=4$ per group) and for plasma proteins and hematocrit at 30 and $60 \mathrm{~min}$ after the onset of restraint ( $n=3$ per group per time point).

Blood samples used for analysis of plasma hormones were collected into chilled tubes containing EDTA and centrifuged at $3500 \mathrm{rpm}$, and those used for determination of plasma proteins and hematocrit were collected into microcapillary tubes and centrifuged at $3000 \mathrm{rpm}$. Samples were stored at $-80^{\circ} \mathrm{C}$ until analysis for hormones via radioimmunoassay (RIA). Plasma proteins and hematocrit were determined using a refractometer and microcapillary reader, respectively.

Water bottles were removed and rats were administered 0.15 or $2.0 \mathrm{M}$ $\mathrm{NaCl}$ as described. One hour later, tail blood samples (250-300 $\mu \mathrm{l})$ were gently taken to determine pre-restraint levels of adrenocorticotropic hormone (ACTH), corticosterone (CORT), and plasma sodium concentration $(\mathrm{pNa})$. These rats were then immediately placed into plastic restrainers, and tail blood samples were collected 15, 30, and $60 \mathrm{~min}$ after the onset of restraint. After the $60 \mathrm{~min}$ sample, the rats were released to their home cage and a final sample was taken at the $120 \mathrm{~min}$ time point. Blood samples used for ACTH and CORT were collected in chilled tubes containing EDTA and centrifuged at $3500 \mathrm{rpm}$. Plasma samples were stored at $-80^{\circ} \mathrm{C}$ until analysis for hormones via RIA. Plasma sodium concentration was determined using a dual-channel flame photometer (Cole-Parmer). Immediately after collection of the $120 \mathrm{~min}$ blood samples, rats were deeply anesthetized with pentobarbital and perfused with isotonic saline followed by $4 \%$ paraformaldehyde. Brains were extracted, placed in $4 \%$ paraformaldehyde overnight, and, on the following day, were cryoprotected in $30 \%$ sucrose.

Radioimmunoassay. Plasma renin activity was measured by RIA using an ${ }^{125}$ I kit from DiaSorin. Plasma ACTH was measured by RIA using an antiserum donated by Dr. William Engeland (University of Minnesota, Minneapolis, MN) and ${ }^{125} \mathrm{I}$-ACTH as tracer (GE Healthcare). Plasma CORT was measured by RIA using a ${ }^{125} \mathrm{I}$ kit from MP Biomedicals. Plasma OT and AVP were measured using ${ }^{125} \mathrm{I}$ kits from Phoenix Pharmaceuticals.

Immunohistochemistry. Brains were cut on a sliding microtome into three separate series of $30 \mu \mathrm{m}$ coronal sections. Double-label immunohistochemistry for OT/c-Fos immunoreactivity or AVP/c-Fos immunoreactivity was completed with free-floating sections, and all rinses consisted of $5 \times 5 \mathrm{~min}$ in $50 \mathrm{~mm}$ potassium PBS. Briefly, sections were rinsed and blocked in $1 \%$ bovine serum albumin with $0.1 \%$ Triton-X for $1 \mathrm{~h}$ and then incubated in primary antibodies overnight. The series double-labeled for OT/c-Fos immunoreactivity was incubated in mouse monoclonal anti-OT (Abcam; ab78364; 1:10,000) and rabbit polyclonal anti-c-Fos (Santa Cruz; 1:3000) overnight. The following day, this series was rinsed and incubated (1:500) with secondary antibodies (Jackson ImmunoResearch; Cy3 anti-mouse; Invitrogen; Alexa 488 anti-rabbit). The series double-labeled for AVP/c-Fos immunoreactivity was first incubated in rabbit anti-c-Fos (Santa Cruz; 1:3000) overnight and the next day was rinsed and incubated at 1:500 with $\mathrm{Cy} 3$ anti-rabbit overnight (Jackson ImmunoResearch). The following day, this tissue was rinsed and incubated with rabbit anti-vasopressin neurophysin antibody (courtesy of Alan Robinson, UCLA, Los Angeles, CA; 1:25,000) overnight and the next day rinsed and incubated at 1:500 with Alexa 488 anti-rabbit (Invitrogen). After the final incubation, all sections were rinsed, mounted onto microscope slides, and coverslipped.

Image capture and analysis. Based on anatomical landmarks described by Paxinos and Watson (1997), images of the PVN and supraoptic nucleus (SON) were collected with a fluorescent microscope (Zeiss Apotome). Black-and-white images were collected, and false color was added. Cell counts were obtained for (1) c-Fos immunoreactivity, (2) OTpositive neurons with c-Fos immunoreactivity, (3) AVP-positive neurons with c-Fos immunoreactivity. For each series, five sections through the PVN or SON were taken and matched among subjects and cell counts were taken unilaterally. The average number of cell counts from each nucleus from each animal was calculated, and group means for each experimental condition were determined. Total c-Fos immunoreactivity was determined by compiling and then averaging cell counts from each series of tissue (i.e., 10 sections per nucleus). A researcher blind to the animal's group identification performed image capture and cell counting. Image analysis was done using NIH ImageJ.

Restraint and telemetric recording of cardiovascular parameters and activity. To obtain continuous recording of mean arterial pressure (MAP), heart rate (HR), heart rate variability (HRV), and activity in conscious, freely moving animals, a cohort of rats was anesthetized with isoflurane and implanted with a radiotelemetry transmitter (TA11PAC40; Data Sciences International). The descending aorta was exposed via an abdominal incision, and a catheter extending from the transmitter capsule was placed into the descending aorta and secured with tissue adhesive (Vetbond; $3 \mathrm{M}$ ) and a cellulose patch. The capsule was sutured to the abdominal musculature, the abdominal musculature was sutured, and wound clips were applied to the skin. After 2 weeks of recovery, cardiovascular parameters were recorded and rats were divided into two groups matched for basal MAP and HR. One week later, cardiovascular parameters and activity were recorded from the rats after they were administered 0.15 or $2.0 \mathrm{M} \mathrm{NaCl}$ and then subjected to 60 min restraint as described above. Specifically, cardiovascular parameters and activity were continuously recorded starting $60 \mathrm{~min}$ before, during, and until 120 min after restraint. Two weeks later, the conditions were reversed, thereby using a within-subjects design with each animal serving as its own control. Mean arterial pressure, HR, HRV, and activity were determined using A.R.T. Platinum software (Data Sciences International). Data for each parameter were averaged into $10 \mathrm{~min}$ bins for each animal in each condition, and subsequently, group means and SEs were calculated. 

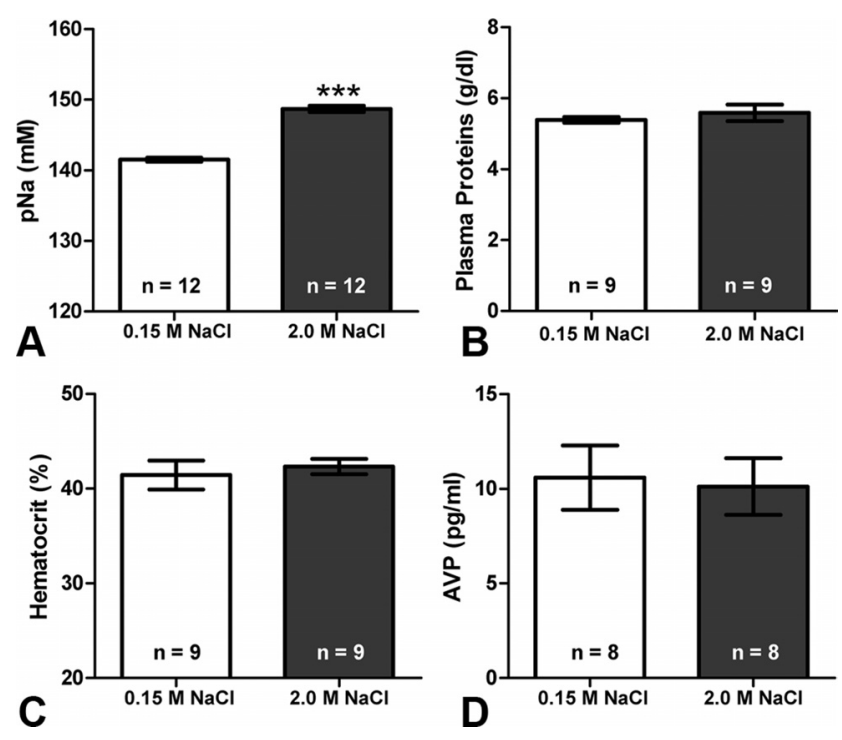

Figure 1. Plasma measurements taken from samples collected before, during and after restraint. Statistical analyses did not detect an effect of time, and consequently, the data are presented as group means. $\boldsymbol{A}-\boldsymbol{D}$, Administration of $2.0 \mathrm{M} \mathrm{NaCl}$ significantly increases pNa relative to controls $(\boldsymbol{A})$, but has no effect on plasma proteins $(\boldsymbol{B})$, hematocrits $(\boldsymbol{C})$, or AVP $(\boldsymbol{D})$. ${ }^{* * *} p<0.01$. Error bars indicate SEM.

Social interaction. A modified version of the social interaction test (SI) (File and Seth, 2003; Shekhar et al., 2006) was used to assess the effect of acute salt loading on anxiety-like behavior. A separate cohort of rats was administered saline as described and $60 \mathrm{~min}$ later were placed into a novel cage with an unfamiliar conspecific of similar size for $10 \mathrm{~min}$. A video camera was fixed above the cage and all behavior was recorded. The total amount of time the experimental rat spent interacting (touching, sniffing, etc.) with the unfamiliar conspecific was subsequently determined by an observer unaware of the treatment conditions. Videos were also scored for aggressive behaviors (biting and lateral attack) as previously described (Nguyen et al., 2007).

Statistical analyses. Data were analyzed using Statistica (StatSoft). All data are expressed as mean \pm SEM. Plasma renin activity, OT, AVP, ACTH, plasma proteins, and hematocrits were analyzed with a twofactor ANOVA with time $(0,15,30,60$, or $120 \mathrm{~min})$ and condition $(0.15$ $\mathrm{M} \mathrm{NaCl}$ or $2 \mathrm{M} \mathrm{NaCl}$ ) as factors. Plasma levels of CORT and sodium were analyzed with two-factor repeated-measures ANOVA with condition and time as factors. Cardiovascular parameters and activity were also analyzed with two-factor repeated-measures ANOVA with condition and time as factors. Area under the curve (AUC) for ACTH and CORT were analyzed with a two-tailed $t$ test. Cell counts for c-Fos immunoreactivity, OT/c-Fos immunoreactivity, and AVP/c-Fos immunoreactivity were analyzed with a one-way ANOVA with condition as the factor. Social interaction and aggressive behavior were assessed with a two-tailed $t$ test. Main effects or interactions $(p<0.05)$ were assessed with a Fisher least significant difference test.

\section{Results}

Osmotic dehydration elevates $\mathrm{pNa}$ but has no effect on plasma proteins, hematocrit, or AVP

Figure 1 depicts the effect of 0.15 or $2.0 \mathrm{M} \mathrm{NaCl}$ administration on $\mathrm{pNa}$, plasma proteins, hematocrit, and plasma AVP. Statistical analyses did not detect an effect of time or a time by condition interaction for $\mathrm{pNa}$, plasma proteins, hematocrit, or AVP, and consequently, the results are presented as group means. As expected, there was an effect of condition $\left(F_{(1,22)}=36.6 ; p<0.01\right)$ on $\mathrm{pNa}$, with rats receiving $2.0 \mathrm{M} \mathrm{NaCl}$ having significantly elevated $\mathrm{pNa}$ compared with controls. However, there was no effect of $2.0 \mathrm{M} \mathrm{NaCl}$ on plasma proteins, hematocrit, or AVP at the postinjection time points examined.
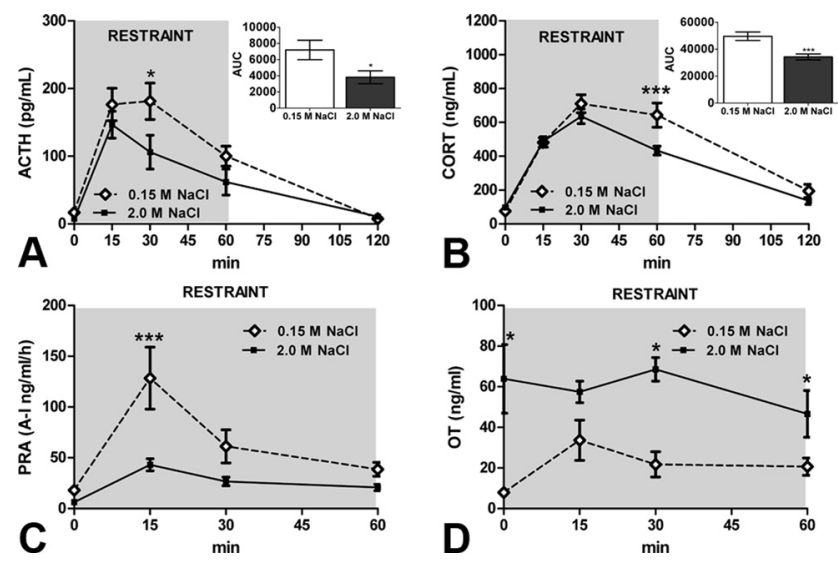

Figure 2. $\quad \boldsymbol{A}$, Restraint elevates $\mathrm{ACTH}$; however, rats treated with $2.0 \mathrm{~m} \mathrm{NaCl}$ have a blunted response compared with controls. $\boldsymbol{B}$, Similarly, restraint increases CORT, but rats treated with $2.0 \mathrm{M} \mathrm{NaCl}$ have decreased CORT relative to controls. C, Restraint significantly increases PRA at 15 min for controls, but a significant increase was not detected in rats given $2.0 \mathrm{~m} \mathrm{NaCl}$. D, Relative to controls, rats injected with $2.0 \mathrm{M} \mathrm{NaCl}$ have increased systemic $0 \mathrm{~T}$ before and 30 and $60 \mathrm{~min}$ after the onset of restraint. ACTH/CORT, $n=12$ per condition; PRA/OT, $n=5-6$ per time point. ${ }^{* * *} p<0.01 ;{ }^{*} p<0.05$. Error bars indicate SEM.

Osmotic dehydration attenuates ACTH, CORT, and PRA, but elevates $\mathrm{OT}$ during restraint

As depicted in Figure 2, restraint increased ACTH $\left(F_{(1,4)}=6.78\right.$; $p<0.05)$ and $\operatorname{CORT}\left(F_{(1,4)}=95.2 ; p<0.05\right)$ above pre-restraint levels. Administration of $2.0 \mathrm{M} \mathrm{NaCl}$ significantly decreased $\operatorname{ACTH}\left(F_{(1,4)}=36.8 ; p<0.05\right)$ and $\operatorname{CORT}\left(\left(F_{(1,22)}=5.36 ; p<\right.\right.$ $0.05)$ relative to controls. Specifically, in rats given $2.0 \mathrm{M} \mathrm{NaCl}$, ACTH was reduced at $30 \mathrm{~min}(p<0.05)$ and CORT at $60 \mathrm{~min}$ $(p<0.01)$, and inhibitory effects of $2.0 \mathrm{M} \mathrm{NaCl}$ were also observed over the entire stress time course (AUC insets; $p<0.05$ ). Osmotic dehydration eliminated $\left(F_{(1,3)}=9.61 ; p<0.001\right)$ the robust increase in PRA that occurred in the control group (Fig. $2 C)$. Consequently, the mean PRA of controls at $15 \mathrm{~min}$ was significantly $\left(F_{(1,3)}=13.7 ; p<0.05\right)$ greater than that of rats receiving injections of $2.0 \mathrm{M} \mathrm{NaCl}$. Contrary to its effects on ACTH, CORT, and PRA, acute osmotic dehydration significantly $\left(F_{(1,3)}=41.6 ; p<0.01\right)$ increased plasma levels of OT when compared with controls (Fig. 2D). Post hoc analyses revealed that, relative to controls, $2.0 \mathrm{M} \mathrm{NaCl}$ significantly increased OT at the pre-restraint time point $(p<0.01)$ as well as at $30 \mathrm{~min}(p<0.05)$ and $60 \min (p<0.01)$ after the onset of restraint.

\section{Osmotic dehydration increases neuronal activation in OT- and AVP-containing neurons}

Figures 3-5 depict the number of cells expressing c-Fos, OT/cFos, or AVP/c-Fos immunoreactivity in the PVN and SON of rats given 0.15 or $2.0 \mathrm{M} \mathrm{NaCl}$ and subsequently subjected to restraint. Although control and experimental rats had similar amounts of c-Fos immunoreactivity within the PVN $\left(F_{(1,18)}=1.64 ; p=\right.$ 0.22 ), treatment with $2.0 \mathrm{M} \mathrm{NaCl}$ significantly increased c-Fos within the $\operatorname{SON}\left(F_{(1,18)}=32.4 ; p<0.001\right)$. Adjacent sections were doubled-labeled for OT/c-Fos or AVP/c-Fos to further examine the phenotype of cells that were activated within the PVN and SON. Rats treated with $2.0 \mathrm{M} \mathrm{NaCl}$ had significantly greater numbers of cells expressing OT/c-Fos within the PVN $\left(F_{(1,13)}=\right.$ $75.7 ; p<0.001)$ and $\operatorname{SON}\left(F_{(1,13)}=85.7 ; p<0.001\right)$ when compared with controls, and this was also true when AVP/c-Fos expression was evaluated $\left(\mathrm{PVN}, F_{(1,13)}=18.7, p<0.001\right.$; SON, $\left.F_{(1,13)}=22.3, p<0.001\right)$. 


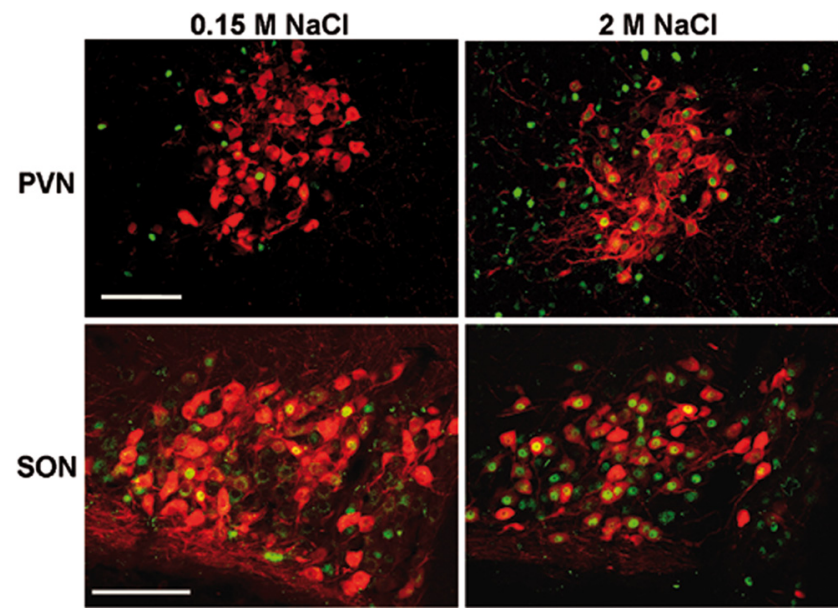

Figure 3. Representative coronal sections through the PVN (top) or SON (bottom) of rats treated with $0.15 \mathrm{~m} \mathrm{NaCl}$ (left) or $2.0 \mathrm{~m} \mathrm{NaCl}$ (right) and then subjected to restraint. Red cell bodies are positive for 0T. Green nuclei are positive for c-Fos. Scale bars, $100 \mu \mathrm{m}$.
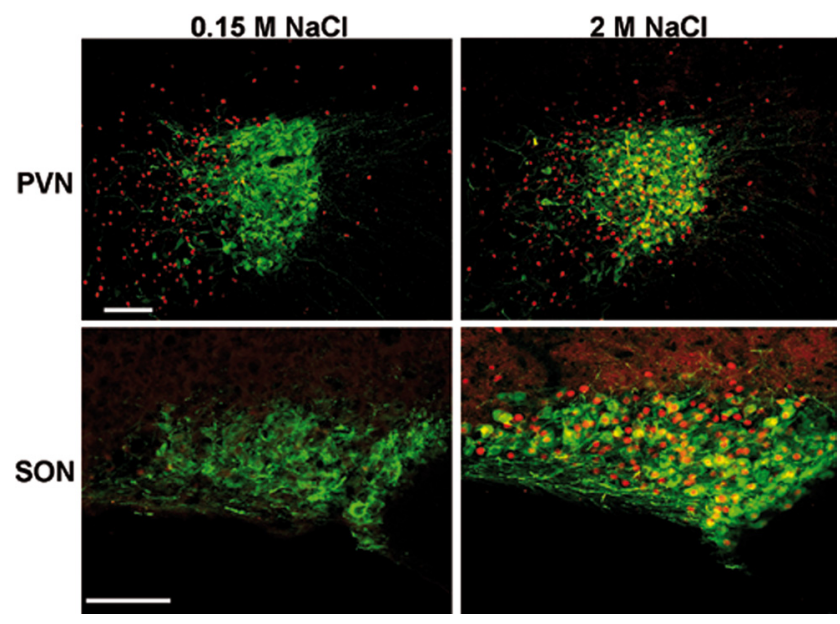

Figure 4. Representative coronal sections through the PVN (top) or SON (bottom) of rats treated with $0.15 \mathrm{~m} \mathrm{NaCl}$ (left) or $2.0 \mathrm{~m} \mathrm{NaCl}$ (right) and then subjected to restraint. Green cell bodies are positive for AVP. Red nuclei are positive for c-Fos. Scale bars, $100 \mu \mathrm{m}$.

\section{PVN}

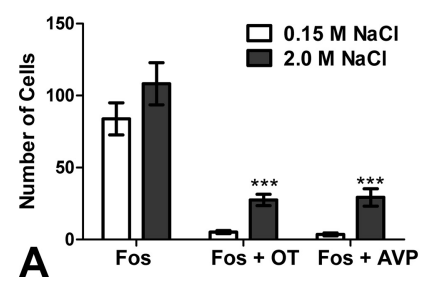

SON

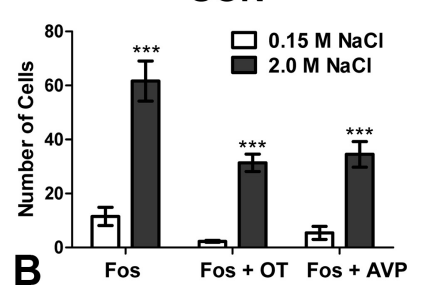

Figure 5. A, Average number of cells within the PVN expressing immunoreactivity for c-Fos, C-Fos/OT, or c-Fos/AVP. B, Average number of cells within the SON expressing immunoreactivity for c-Fos, c-Fos/0T, or c-Fos/AVP $(n=6-9) .{ }^{* *} p<0.01$. Error bars indicate SEM.

\section{Osmotic dehydration attenuates the cardiovascular response} to restraint

Figure 6 depicts the MAP, HR, HRV, and activity of rats administered 0.15 or $2.0 \mathrm{M} \mathrm{NaCl} 1 \mathrm{~h}$ before a 60 min restraint. MAP was significantly elevated $\left(F_{(1,24)}=35.3 ; p<0.001\right)$ across time and this interacted with experimental condition $\left(F_{(1,24)}=2.03 ; p<\right.$ 0.01 ). Rats given $0.15 \mathrm{M} \mathrm{NaCl}$ had significantly increased MAP $(p<0.05)$ relative to baseline at $-50,-40,-30$, and $-20 \mathrm{~min}$,
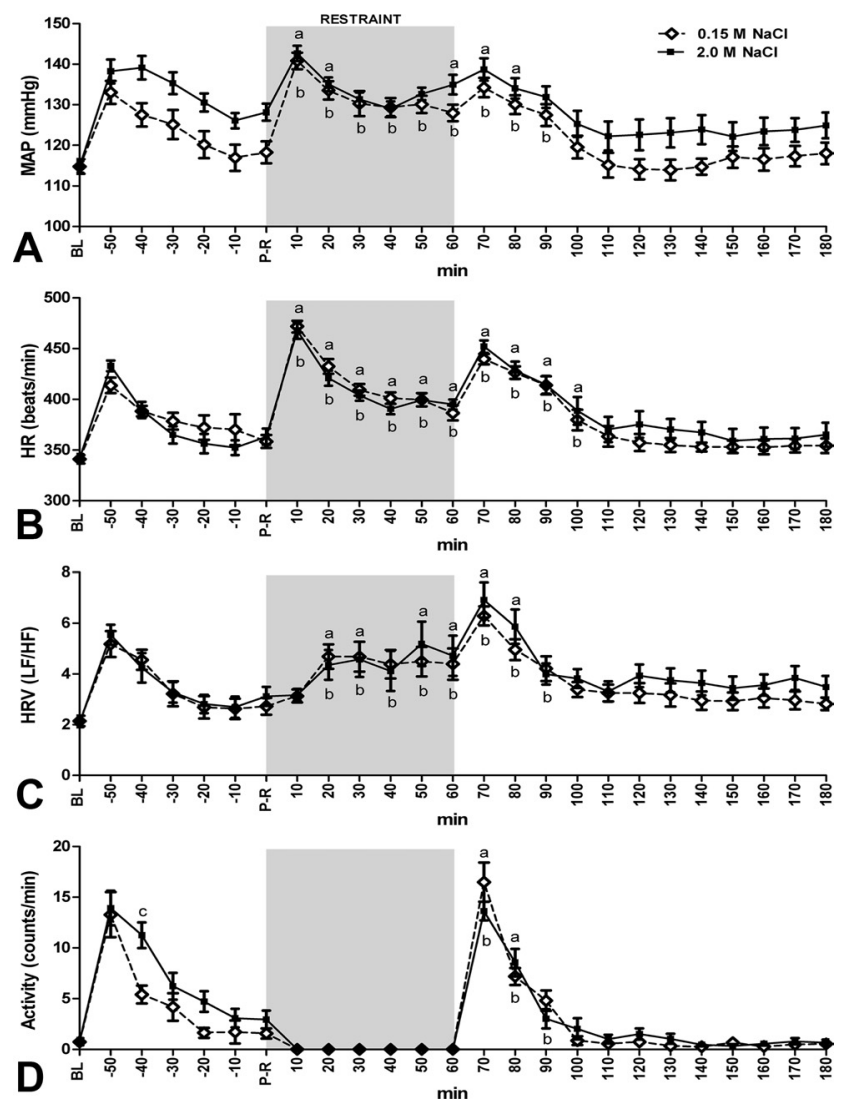

Figure 6. A, Relative to P-R, restraint significantly increased MAP of control rats until 100 min; however, the MAP of rats given $2.0 \mathrm{M} \mathrm{NaCl}$ returns to P-R levels at 30 min during restraint and ultimately recovers at $90 \mathrm{~min}$. $\boldsymbol{B}$, The HR response to restraint is similar in rats given $0.15 \mathrm{M}$ $\mathrm{NaCl}$ or $2 \mathrm{M} \mathrm{NaCl}$. C, Twenty minutes after the onset of restraint, the LF/HF ratio is elevated above $\mathrm{P}$-R levels and remains elevated at $90 \mathrm{~min}$ for controls. In contrast, LF/HF ratio of rats given 2.0 $M \mathrm{NaCl}$ returns to $P-R$ at $40 \mathrm{~min}$ during restraint and ultimately recovers at $80 \mathrm{~min}$. D, Administration of $2.0 \mathrm{~m} \mathrm{NaCl}$ significantly increases activity at $-40 \mathrm{~min}$ when compared with that of controls, but both groups return to BL before restraint. After release from the restrainer the activity of control rats recovers to $P-R$ at $100 \mathrm{~min}$. After release, the activity of rats given $2.0 \mathrm{M}$ $\mathrm{NaCl}$ recovers to $\mathrm{P}-\mathrm{R}$ more quickly at $90 \mathrm{~min}$. BL, Baseline; $\mathrm{P}-\mathrm{R}$, pre-restraint. a, $2.0 \mathrm{~m} \mathrm{NaCl}$ different from $P-R ; b, 0.15 \mathrm{~m} \mathrm{NaCl}$ different from P-R; C, $2.0 \mathrm{M} \mathrm{NaCl}$ different from $0.15 \mathrm{~m} \mathrm{NaCl}$; $n=13$ per condition; significant differences, $p<0.05$. Error bars indicate SEM.

but recovered to basal levels at $-10 \mathrm{~min}$ or before the onset of restraint. In contrast, administration of $2.0 \mathrm{M} \mathrm{NaCl}$ significantly elevated $(p<0.05)$ MAP above baseline at all subsequent measures. Restraint significantly $(p<0.05)$ elevated MAP relative to the pre-restraint time point at 10, 20, 30, 40, 50, 60, 70, 80, and 90 min for control rats. After restraint, rats subjected to osmotic dehydration recovered more quickly than did controls, with MAP of rats given $2.0 \mathrm{M} \mathrm{NaCl}$ returning to pre-restraint levels at $30 \mathrm{~min}$. Although the MAP of rats given $2.0 \mathrm{M} \mathrm{NaCl}$ was significantly elevated $(p<0.05)$ at 60,70 , and 80 min after release from the restrainer, MAP returned to pre-restraint values by $90 \mathrm{~min}$, or 10 min sooner than controls (Fig. 5A).

Heart rate also differed significantly $\left(F_{(1,24)}=61.7 ; p<0.01\right)$ over time, and this interacted with the experimental condition $\left(F_{(1,24)}=1.57 ; p<0.05\right)$. Control injections of $0.15 \mathrm{M} \mathrm{NaCl}$ significantly increased $(p<0.05) \mathrm{HR}$ above baseline until 110 min after the onset of restraint, and restraint significantly elevated $(p<0.05) \mathrm{HR}$ above pre-restraint values until $110 \mathrm{~min}$ (Fig. 5B). Conversely, $2.0 \mathrm{M} \mathrm{NaCl}$ significantly increased $(p<$ $0.05) \mathrm{HR}$ above baseline at $-50,-40$, and $-30 \mathrm{~min}$, but it returned to basal levels at $-20 \mathrm{~min}$. Similar to controls, rats 
given $2.0 \mathrm{M} \mathrm{NaCl}$ had HR return $(p<0.05)$ to pre-restraint levels at $110 \mathrm{~min}$.

To examine the effects of osmotic dehydration and restraint on autonomic tone, we examined HRV [as determined by the ratio of low frequency/high frequency spectra (LF/HF)]. Larger values are indicative of increased sympathetic outflow and parasympathetic withdrawal (Malik, 1996). As depicted in Figure 6C, the LF/HF ratio significantly increased over time $\left(F_{(1,24)}=14.9\right.$; $p<0.01)$. Post hoc analyses revealed that administration of $0.15 \mathrm{M}$ $\mathrm{NaCl}$ increased $(p<0.05)$ the LF/HF ratio at $-50,-40$, and -30 min, and restraint significantly increased $(p<0.05)$ the ratio at $20,30,40,50$, and 60 min during and 70, 80, and 90 min after restraint. The LF/HF ratio was significantly increased for rats given $2.0 \mathrm{M} \mathrm{NaCl}(p<0.05)$ at $-50,-40$, and $-30 \mathrm{~min}$; however, the LF/HF ratio returned to pre-restraint levels at $40 \mathrm{~min}$ during the restraint and after release returned to pre-restraint levels at $80 \mathrm{~min}$, which was 10 min more quickly than occurred in controls. Recent studies demonstrate that the LF/HF ratio is elevated in subjects delivered hypertonic saline subcutaneously, and this effect is attributed to the pain that accompanies the injection (Burton et al., 2009). In our study, the LF/HF ratio of rats given 0.15 or $2.0 \mathrm{M} \mathrm{NaCl}$ were similar before restraint and vocalizations subsequent to saline administration were not observed, which suggests that pretreatment with lidocaine effectively decreased the pain associated with subcutaneous administration of hypertonic saline.

There were effects of time $\left(F_{(1,6)}=34.8 ; p<0.05\right)$ and condition $\left(F_{(1,6)}=6.51 ; p<0.05\right)$ on the activity (horizontal counts per minute) that followed injection of 0.15 or $2.0 \mathrm{M} \mathrm{NaCl}$. Control rats had significantly increased activity $(p<0.05)$ relative to baseline at $-50,-40$, and $-30 \mathrm{~min}$, and after these rats were released from the restrainers, activity significantly increased ( $p<$ $0.05)$ relative to pre-restraint levels at 70,80 , and $90 \mathrm{~min}$. Rats given $2.0 \mathrm{M} \mathrm{NaCl}$ had significantly increased activity $(p<0.05)$ relative to baseline at $-50,-40,-30$, and $-20 \mathrm{~min}$, and the activity at -40 min was significantly greater $(p<0.05)$ than that of controls. After osmotically dehydrated rats were released from the restrainers, activity significantly increased $(p<0.05)$ relative to pre-restraint levels, but recovered by $90 \mathrm{~min}$, which is $10 \mathrm{~min}$ sooner than it did for controls.

\section{Osmotic dehydration promotes social interactions in a paradigm used to assess anxiety-like behavior}

We used the social interaction paradigm to determine whether the attenuated HPA and cardiovascular responses of osmotically dehydrated rats was predictive of decreased anxiety-like behavior. Rats that were treated with $2.0 \mathrm{M} \mathrm{NaCl}$ before initiation of the social interaction test spent significantly more time engaging unfamiliar conspecifics than did control rats, suggesting that acute osmotic dehydration is anxiolytic and enhances social behavior $(65.8 \pm 2.37$ vs $73.4 \pm 2.13 ; n=9-10 ; p<0.05)$. Analysis of aggressive behavior found no occurrences of biting or lateral attacks. Consequently, videos were scored for more subtle forms of aggression (on top of, following close), but the number of these behaviors were similar ( $p=0.24)$ between osmotically dehydrated $(2.2 \pm 0.85)$ and control $(1 \pm 0.41)$ groups.

\section{Discussion}

The present series of experiments provides novel evidence for reduced responding to psychogenic stressors by an acute physical challenge (hypernatremia). Rats rendered mildly hypernatremic had decreased HPA activation in response to physical restraint, a model of psychogenic stress. Comparably treated rats had ele- vated MAP, and this was associated with a blunted restraintinduced increase in MAP and faster post-restraint recovery. Hypernatremia also eliminated the restraint-induced increase of PRA, but elevated circulating OT and decreased anxiety-like behavior in a social situation. In sum, acute hypernatremia potently and simultaneously inhibited the HPA, cardiovascular, and behavioral limbs of the stress response.

Whether these stress-dampening effects are specific to hypernatremia or generalize to other challenges warrants some discussion. In this regard, another homeostatic challenge, caloric restriction, elevated basal and restraint-induced CORT, but attenuated the cardiovascular response to restraint by promoting faster recovery to resting heart rate (Flak et al., 2011). These results indicate that caloric restriction and osmotic dehydration differentially mediate restraint-induced activation of the HPA axis, but both homeostatic challenges influence cardiovascular responding to restraint with caloric restriction affecting $\mathrm{HR}$ and osmotic dehydration affecting blood pressure. Together, these results suggest that all homeostatic challenges do not similarly affect subsequent responding to psychogenic stressors.

In the present study, administration of hypertonic saline significantly increased $\mathrm{pNa}$ relative to controls but did not affect indices of blood volume (i.e., plasma proteins and hematocrit) or circulating levels of AVP. At first glance, these results are in conflict with those demonstrating that administration of hypertonic saline produces dynamic changes in blood volume and circulating levels of AVP (Stricker and Verbalis, 1986; Taylor et al., 2008). However, the effects of acute salt loading on AVP are dependent on the rate of increase in plasma osmolality as well as the maximal osmolality attained (Robertson, 1976). In this regard, the largest increases in pNa occur 15-30 min after administration of hypertonic saline and the greatest elevations in circulating AVP are observed at these times as well (Stricker and Verbalis, 1986). Our plasma measures were taken $60,75,90$, and 120 min after administration of $2 \mathrm{M} \mathrm{NaCl}$, and at these times $\mathrm{pNa}$ and AVP have fallen dramatically (Stricker and Verbalis, 1986). Consequently, it is likely that we did not detect a significant increase in circulating AVP because our measurements were taken when the effects of hypernatremia on AVP were approaching nadir. Timing likely also influenced our indices of blood volume, and consistent with this, another study that took samples 60 min after delivery of isotonic or hypertonic saline found no differences in plasma proteins and hematocrits (Curtis et al., 2004). Thus, our experiments were conducted subsequent to the dynamic effects of acute hypernatremia, minimizing the potential influence that such changes might have on stress responsiveness.

Activation of the HPA axis is a hallmark metric for assessing stress responding (for review, see de Kloet, 2008). Real or perceived threats to an organism trigger activation of the HPA axis, which increases circulating ACTH and CORT. Because hydroelectrolyte imbalance affects most physiological processes, it was unexpected that hypernatremia would attenuate ACTH and CORT release following psychogenic stress. Reduced ACTH implies a central inhibition of the HPA axis, and consistent with this, chronic salt loading decreases nonstressed levels of plasma ACTH and corticotrophin-releasing hormone $(\mathrm{CRH})$ within the parvocellular PVN (Lightman and Young, 1987) and reduces elevations in ACTH and CRH after immobilization stress (Amaya et al., 2001).

Interestingly, hyperosmolality decreases CRH mRNA within parvocellular neurons associated with HPA activation, but increases CRH mRNA within magnocellular neurons regulating hydromineral balance (Young, 1986). Because of these canceling 
effects, the total levels of CRH mRNA within the PVN are unaffected by osmotic dehydration. In our study, hypernatremia increased the activation of magnocellular OT- and AVP-containing neurons after restraint; however, the total number of cells expressing c-Fos within the PVN was not different between the osmotically dehydrated and control groups. This suggests that the effects of hypernatremia and restraint on activation of the PVN are not additive. Rather, osmotic dehydration activates OTand AVP-containing neurons, but inhibits activation of parvocellular CRH-containing neurons in the PVN, thereby blunting the HPA response to psychogenic stress.

Stress exposure also shifts autonomic balance to favor increased sympathetic outflow and parasympathetic withdrawal, manifested physiologically as elevated MAP and HR. Consistent with previous studies, hypertonic saline significantly increased MAP, an effect believed to be mediated by AVP and the sympathetic nervous system (Garcia-Estañ et al., 1989; Antunes et al., 2006). Because the MAP of the hypernatremic rats was elevated at the initiation of stress exposure, the increase in $\operatorname{MAP}(\Delta)$ relative to the pre-restraint time point was attenuated, and recovered to pre-restraint levels more quickly than normonatremic controls. One interpretation of these results is that osmotic dehydration resets basal MAP to higher levels before stress exposure, and this buffers against the large deviations in MAP (i.e., promotes smaller $\Delta$ and quicker recovery) that occur when animals are challenged with stressors.

In contrast to MAP, the HR, HRV, and activity of hypernatremic rats returned to baseline by the onset of restraint. Heart rate variability, indicated by the ratio of LF/HF spectra, was increased by restraint for both control and osmotically dehydrated rats, implying that this model of psychogenic stress is accompanied by augmented sympathetic tone and/or vagal withdrawal. The more rapid return of the LF/HF ratio to pre-restraint levels in hypernatremic rats suggests that acute osmotic dehydration promotes the restoration of sympatho-vagal balance during and after stress exposure. Hypernatremic rats also returned to behavioral quiescence (low activity) more quickly than controls after stress exposure. Collectively, these results suggest that acute osmotic dehydration buffers increases in MAP that accompany stress challenges and promotes quicker recovery, possibly by reducing sympathetic tone and enhancing vagal recovery.

To determine whether a previous stressor can influence behavior, we evaluated performance in the social interaction test, an experimentally validated paradigm used to assess anxiety-like behavior in rodents (File and Hyde, 1978; File, 1980). Consistent with our metrics of HPA and cardiovascular responding, rats rendered mildly hypernatremic spent more time interacting with unfamiliar conspecifics relative to normonatremic controls, suggesting that acute osmotic dehydration decreases anxiety-like behavior and promotes social interactions.

\section{Conclusions}

In sum, acute hypernatremia limited responding to psychogenic stressors across several levels of analyses. In our study, psychogenic stress produced a robust increase in PRA, an index of circulating angiotensin II (ANGII), which was completely abolished by hypernatremia. Conventionally, ANGII is thought of as a mediator of body fluid homeostasis and salt loading suppresses PRA to reduce the adrenal secretion of aldosterone and this inhibits renal sodium reabsorption (Husain et al., 1987). However, emerging evidence suggests that ANGII is a potently acting "stress hormone" (for review, see Saavedra et al., 2005; Saavedra and Benicky, 2007). In this regard, recent studies demonstrate that ANGII acts on systemic and/or central angiotensin type 1 receptors to activate the HPA axis (Krause et al., 2008), facilitate the cardiovascular response to stress exposure (Kubo et al., 2001; Davern et al., 2009), and promote anxiety-like behavior (Pavel et al., 2008). Thus, as hypernatremia suppresses the synthesis of ANGII to alleviate hydromineral imbalance, it concurrently decreases responsiveness to psychogenic stressors.

Contrary to the effects on PRA, acute osmotic dehydration greatly elevated circulating levels of OT, which correspond with the robust activation of OT-containing neurons in the PVN and SON. These results concur with seminal studies of Stricker and Verbalis (Stricker and Verbalis, 1986; Rinaman et al., 1997) demonstrating that hypernatremia activates hypothalamic oxytocinergic neurons, thereby elevating circulating levels of this hormone. Studies from this group established that during hypernatremia OT acts systemically to promote natriuresis (Verbalis et al., 1991) and centrally to inhibit salt appetite (Blackburn et al., 1993). Although the effects of central and systemic OT on hydromineral balance are well documented, OT is also a powerful mediator of stress responding and social behaviors (for review, see Neumann, 2002, 2007; Amico et al., 2004; Carter et al., 2008; Donaldson and Young, 2008). That is, OT is found to blunt restraint-induced HPA activation (Windle et al., 1997, 2004), decrease cardiovascular responding to isolation stress (Grippo et al., 2009), reduce anxiety-like behavior (Windle et al., 1997), and promote social interactions (Lee et al., 2005). The mechanism underlying these effects is an active area of research, but accumulating evidence suggests OT released from the SON and PVN acts within hypothalamic and extra-hypothalamic nuclei to influence stress responding and mood (for review, see Ludwig and Pittman, 2003; Neumann, 2007). Although some stress paradigms trigger the release of OT from the SON and PVN (Engelmann and Ludwig, 2004), systemic hypernatremia reliably augments central levels of this peptide (Ludwig et al., 1994). We examined stress responding $1 \mathrm{~h}$ after administration of hypertonic saline, when the central release of OT is greatly elevated (Ludwig et al., 1994). Therefore, it is likely that, subsequent to hypernatremia, central OT was increased and is a physiologically relevant response that blunts stress responding and promotes social behavior when challenged with dehydration.

The current results, in agreement with previous studies, strongly suggest that acute hypernatremia limits stress responding by abolishing the augmentation in PRA that otherwise accompanies stress exposure and by elevating central oxytocinergic activity. In essence, hypernatremia may limit generalized stress responding by suppressing the release of the "prostress hormone," ANGII, while increasing activity of OT, an "antistress hormone."

\section{Perspectives from the watering hole}

Hypernatremia activates mechanisms that alleviate increased plasma tonicity while limiting responses to psychogenic stressors and promoting social behavior. It is possible that the same system that evolved to maintain hydromineral balance when faced with hypernatremia also suppresses the fear and anxiety associated with approaching a communal water source, while perhaps introducing tolerance to fearful stimuli (i.e., conspecifics or potential presence of predators) that may be encountered when engaging in drinking behavior. Our data suggest an integrated neural mechanism that links bodily feedback to a spectrum of responses that promote alleviation of physical needs. 


\section{References}

Amaya F, Tanaka M, Hayashi S, Tanaka Y, Ibata Y (2001) Hypothalamopituitary-adrenal axis sensitization after chronic salt loading. Neuroendocrinology 73:185-193.

Amico JA, Mantella RC, Vollmer RR, Li X (2004) Anxiety and stress responses in female oxytocin deficient mice. J Neuroendocrinol 16 : 319-324.

Antunes VR, Yao ST, Pickering AE, Murphy D, Paton JF (2006) A spinal vasopressinergic mechanism mediates hyperosmolality-induced sympathoexcitation. J Physiol 576:569-583.

Blackburn RE, Samson WK, Fulton RJ, Stricker EM, Verbalis JG (1993) Central oxytocin inhibition of salt appetite in rats: evidence for differential sensing of plasma sodium and osmolality. Proc Natl Acad Sci U S A 90:10380-10384.

Bourque CW (2008) Central mechanisms of osmosensation and systemic osmoregulation. Nat Rev Neurosci 9:519-531.

Burton AR, Birznieks I, Bolton PS, Henderson LA, Macefield VG (2009) Effects of deep and superficial experimentally induced acute pain on muscle sympathetic nerve activity in human subjects. J Physiol 587:183-193.

Carter CS, Grippo AJ, Pournajafi-Nazarloo H, Ruscio MG, Porges SW (2008) Oxytocin, vasopressin and sociality. Prog Brain Res 170:331-336.

Chotiwat C, Harris RB (2006) Increased anxiety-like behavior during the post-stress period in mice exposed to repeated restraint stress. Horm Behav 50:489-495.

Curtis KS, Krause EG, Wong DL, Contreras RJ (2004) Gestational and early postnatal dietary $\mathrm{NaCl}$ levels affect $\mathrm{NaCl}$ intake, but not stimulated water intake, by adult rats. Am J Physiol Regul Integr Comp Physiol 286: R1043-R1050.

Davern PJ, Chen D, Head GA, Chavez CA, Walther T, Mayorov DN (2009) Role of angiotensin II type 1A receptors in cardiovascular reactivity and neuronal activation after aversive stress in mice. Hypertension 54: $1262-1268$.

de Kloet ER (2008) About stress hormones and resilience to psychopathology. J Neuroendocrinol 20:885-892.

Donaldson ZR, Young LJ (2008) Oxytocin, vasopressin, and the neurogenetics of sociality. Science 322:900-904.

Engelmann M, Ludwig M (2004) The activity of the hypothalamoneurohypophysial system in response to acute stressor exposure: neuroendocrine and electrophysiological observations. Stress 7:91-96.

File SE (1980) The use of social interaction as a method for detecting anxiolytic activity of chlordiazepoxide-like drugs. J Neurosci Methods 2:219-238.

File SE, Hyde JR (1978) Can social interaction be used to measure anxiety? Br J Pharmacol 62:19-24.

File SE, Seth P (2003) A review of 25 years of the social interaction test. Eur J Pharmacol 463:35-53.

Flak JN, Jankord RJ, Solomon MB, Krause EG, Herman JP (2011) Opposing effects of chronic stress and weight restriction on cardiovascular, neuroendocrine, and metabolic function. Physiol Behav. Advance online publication. Retrieved March 16, 2011. doi:10.1016/j.physbeh.2011.03.002.

Garcia-Estañ J, Carbonell LF, Garcia-Salom M, Salazar FJ, Quesada T (1989) Hemodynamic effects of hypertonic saline in the conscious rat. Life Sci 44:1343-1350.

Grippo AJ, Trahanas DM, Zimmerman RR 2nd, Porges SW, Carter CS (2009) Oxytocin protects against negative behavioral and autonomic consequences of long-term social isolation. Psychoneuroendocrinology 34:1542-1553.

Husain A, DeSilva P, Speth RC, Bumpus FM (1987) Regulation of angiotensin II in rat adrenal gland. Circ Res 60:640-648.

Krause EG, Melhorn SJ, Davis JF, Scott KA, Ma LY, de Kloet AD, Benoit SC, Woods SC, Sakai RR (2008) Angiotensin type 1 receptors in the subfornical organ mediate the drinking and hypothalamic-pituitary-adrenal response to systemic isoproterenol. Endocrinology 149:6416-6424.

Kubo T, Numakura H, Endo S, Hagiwara Y, Fukumori R (2001) Angiotensin receptor blockade in the anterior hypothalamic area inhibits stressinduced pressor responses in rats. Brain Res Bull 56:569-574.

Lee PR, Brady DL, Shapiro RA, Dorsa DM, Koenig JI (2005) Social interac- tion deficits caused by chronic phencyclidine administration are reversed by oxytocin. Neuropsychopharmacology 30:1883-1894.

Lightman SL, Young WS 3rd (1987) Vasopressin, oxytocin, dynorphin, enkephalin and corticotrophin-releasing factor mRNA stimulation in the rat. J Physiol 394:23-39.

Ludwig M, Pittman QJ (2003) Talking back: dendritic neurotransmitter release. Trends Neurosci 26:255-261.

Ludwig M, Callahan MF, Neumann I, Landgraf R, Morris M (1994) Systemic osmotic stimulation increases vasopressin and oxytocin release within the supraoptic nucleus. J Neuroendocrinol 6:369-373.

Malik M (1996) Heart rate variability: standards of measurement, physiological interpretation, and clinical use. Circulation 93:1043-1065.

Neumann ID (2002) Involvement of the brain oxytocin system in stress coping: interactions with the hypothalamo-pituitary-adrenal axis. Prog Brain Res 139:147-162.

Neumann ID (2007) Stimuli and consequences of dendritic release of oxytocin within the brain. Biochem Soc Trans 35:1252-1257.

Nguyen MM, Tamashiro KL, Melhorn SJ, Ma LY, Gardner SR, Sakai RR (2007) Androgenic influences on behavior, body weight, and body composition in a model of chronic social stress. Endocrinology 148:6145-6156.

Pavel J, Benicky J, Murakami Y, Sanchez-Lemus E, Saavedra JM (2008) Peripherally administered angiotensin II AT1 receptor antagonists are antistress compounds in vivo. Ann N Y Acad Sci 1148:360-366.

Paxinos G, Watson C (1997) The rat brain in stereotaxic coordinates. San Diego: Academic.

Rinaman L, Stricker EM, Hoffman GE, Verbalis JG (1997) Central c-Fos expression in neonatal and adult rats after subcutaneous injection of hypertonic saline. Neuroscience 79:1165-1175.

Robertson GL (1976) The regulation of vasopressin function in health and disease. Recent Prog Horm Res 33:333-385.

Saavedra JM, Benicky J (2007) Brain and peripheral angiotensin II play a major role in stress. Stress 10:185-193.

Saavedra JM, Ando H, Armando I, Baiardi G, Bregonzio C, Juorio A, Macova M (2005) Anti-stress and anti-anxiety effects of centrally acting angiotensin II AT1 receptor antagonists. Regul Pept 128:227-238.

Sgoifo A, Koolhaas JM, Musso E, De Boer SF (1999) Different sympathovagal modulation of heart rate during social and nonsocial stress episodes in wild-type rats. Physiol Behav 67:733-738.

Shekhar A, Johnson PL, Sajdyk TJ, Fitz SD, Keim SR, Kelley PE, Gehlert DR, DiMicco JA (2006) Angiotensin-II is a putative neurotransmitter in lactate-induced panic-like responses in rats with disruption of GABAergic inhibition in the dorsomedial hypothalamus. J Neurosci 26:9205-9215.

Stricker EM, Sved AF (2000) Thirst. Nutrition 16:821-826.

Stricker EM, Verbalis JG (1986) Interaction of osmotic and volume stimuli in regulation of neurohypophyseal secretion in rats. Am J Physiol 250:R267-R275.

Taylor AC, McCarthy JJ, Stocker SD (2008) Mice lacking the transient receptor vanilloid potential 1 channel display normal thirst responses and central Fos activation to hypernatremia. Am J Physiol Regul Integr Comp Physiol 294:R1285-R1293.

Toney GM, Stocker SD (2010) Hyperosmotic activation of CNS sympathetic drive: implications for cardiovascular disease. J Physiol 588: 3375-3384.

Ulrich-Lai YM, Herman JP (2009) Neural regulation of endocrine and autonomic stress responses. Nat Rev Neurosci 10:397-409.

Verbalis JG, Mangione MP, Stricker EM (1991) Oxytocin produces natriuresis in rats at physiological plasma concentrations. Endocrinology 128:1317-1322.

Windle RJ, Shanks N, Lightman SL, Ingram CD (1997) Central oxytocin administration reduces stress-induced corticosterone release and anxiety behavior in rats. Endocrinology 138:2829-2834.

Windle RJ, Kershaw YM, Shanks N, Wood SA, Lightman SL, Ingram CD (2004) Oxytocin attenuates stress-induced c-fos mRNA expression in specific forebrain regions associated with modulation of hypothalamopituitary-adrenal activity. J Neurosci 24:2974-2982.

Young WS 3rd (1986) Corticotropin-releasing factor mRNA in the hypothalamus is affected differently by drinking saline and by dehydration. FEBS Lett 208:158-162. 